\title{
Targeted inhibition of endothelial calpain delays wound healing by reducing inflammation and angiogenesis
}

\author{
Chenlong Yi ${ }^{1}$, Weihua $\mathrm{Wu}^{2}$, Dong Zheng ${ }^{2}$, Guangying Peng ${ }^{1}$, Haoyue Huang ${ }^{1}$, Zhenya Shen ${ }^{1}$ and Xiaomei Teng ${ }^{1}$
}

\begin{abstract}
Wound healing is a multistep phenomenon that relies on complex interactions between various cell types. Calpains are a well-known family of calcium-dependent cysteine proteases that regulate several processes, including cellular adhesion, proliferation, and migration, as well as inflammation and angiogenesis. CAPNS1, the common regulatory subunit of Calpain-1 and 2, is indispensable for catalytic subunit stabilization and activity. Calpain inhibition has been shown to reduce organ damage in various disease models. Here, we report that endothelial calpain-1/2 is crucially involved in skin wound healing. Using a mouse genetic model where Capns1 is deleted only in endothelial cells, we showed that calpain-1/2 disruption is associated with reduced injury-activated inflammation, reduced $\mathrm{CD} 31^{+}$blood vessel density, and delayed wound healing. Moreover, in cultured HUVECs, inhibition of calpain reduced TNF-ainduced proliferation, migration, and tube formation. Deletion of Capns 1 was associated with elevated levels of $1 \mathrm{kB}$ and downregulation of $\beta$-catenin expression in endothelial cells. These observations delineate a novel mechanistic role for calpain in the crosstalk between inflammation and angiogenesis during skin repair.
\end{abstract}

\section{Introduction}

The skin is the largest organ of the human body and acts as a protective barrier against external stimuli and pathogen invasion. In most cases, acute injuries initiate an immediate emergency response to establish a repair and regeneration environment ${ }^{1}$. Skin wound healing undergoes multiple overlapping phases, including a peak angiogenic response after injury, followed by decreased vessel numbers upon wound closure and simultaneously fibrosis and scarring ${ }^{2}$. These repair mechanisms involve in elaborate and intricate processes and remain incompletely understood ${ }^{3}$.

Correspondence: Zhenya Shen (uuzyshen@aliyun.com) or

Xiaomei Teng (tengxiaomei@suda.edu.cn)

${ }^{1}$ Department of Cardiovascular Surgery of the First Affiliated Hospital \&

Institute for Cardiovascular Science, Soochow University, 215006 Suzhou, China

${ }^{2}$ Center of Clinical Laboratory, The First Affiliated Hospital of Soochow

University, 215006 Suzhou, China

These authors contributed equally: Chenlong Yi, Weihua Wu, Dong Zheng

Edited by B. Zhivotovsky
Calcium serves as a modulator in the normal homeostasis of mammalian skin. In wound repair, calcium is predominantly involved as Factor IV in the hemostatic phase, but it is indispensable in cell migration and regeneration patterns in later stages of healing ${ }^{4}$. Calpains belong to a family of calcium-dependent cysteine proteases ${ }^{5}$, which have the unique property of modifying the activity/function of their substrates by protein cleavage. Among 16 family members, calpain- 1 and calpain- 2 are two most extensively studied isoforms, and are expressed ubiquitously in mammals and many other organisms. Of note, calpain-1 and calpain-2 are the only known calpain isoforms expressed in endothelial cells (ECs) ${ }^{6}$. Calpain-1/2 isoforms are heterodimers consisting of CAPN1 and CAPN2 catalytic subunits, respectively, and the common small regulatory subunit CAPNS1, also known as CAPN4. These ubiquitous classical calpain isoforms are regulated by the endogenous calpainspecific inhibitor, calpastatin (CAST). Inhibiting calpain activity has been shown to reduces tissue damage in experimental models of inflammatory diseases ${ }^{7}$, cancer 
progression $^{8-10}$, neurodegeneration ${ }^{11}$, and cardiovascular diseases ${ }^{12}$. Calpain activity also appears to be essential in skin wound healing ${ }^{13}$. Loss-of-function mutations in calpastatin cause PLACK syndrome, which is characterized by multiple skin pathologies ${ }^{14}$. CAST overexpression strikingly delays wound healing with reduced proliferation and reepithelialization ${ }^{13}$. Whereas selective inhibition of calpain-1 in diabetes reverses dermal defects and improves the quality of diabetic skin after wound healing ${ }^{15}$.

Skin wound healing is a complex process. The wound site is usually subject to hypoxic conditions during healing, which results in sprouting angiogenesis. This event is crucial for proliferating tissues and creating an access route for inflammatory cells. In vascular endothelial cells, calpains have been reported to be associated with sprouting angiogenesis ${ }^{16}$. We reported that inhibition of calpain reduced oxidative stress and attenuated endothelial dysfunction in diabetes ${ }^{17}$. Endothelial cell-specific deletion of Capns1 reduced diabetic cardiomyopathy by improving angiogenesis ${ }^{18}$. These findings indicate a critical role of endothelial calpain in microvascular endothelial dysfunction. However, these causes of endothelial dysfunction that delays skin wound healing remain incompletely understood. Calpain activation has been implicated in endothelial dysfunction and inflammation. This raises an intriguing possibility that calpain-mediated endothelial injury may contribute to skin wound healing.

In this study, we investigated the role of endothelial calpain in skin wound healing using mice with endothelial cell-specific deletion of Capns1.

\section{Results}

Deficiency of endothelial cell Capns 1 delays wound healing of dorsal skin

To evaluate the effects of endothelial cell-specific calpain-1/2 disruption following skin injury, full-thickness $15 \mathrm{~mm}$ diameter excisional wounds were made on TEK$\mathrm{CRE}^{+/-}$Capns1 $^{\mathrm{PZ} / \mathrm{PZ}}$ (KO) mice and their wild-type (WT) littermates, and the wounds were monitored for 7 days. Although the dynamics of wound contraction can be variable, as previously described ${ }^{19,20}$, we found a significant delay in wound healing of $\mathrm{KO}$ mice compared with that of WT mice (Fig. 1a). A trend toward reduced wound closure in $\mathrm{KO}$ mice became apparent at 3 days and was significantly different by 6 days (Fig. 1b). The mean wound areas of WT mice were reduced to $28.48 \%$ of the original wound size at 7 days, while those of $\mathrm{KO}$ mice remained at $45.44 \%$ of the original injured areas (Fig. 1b). Morphological abnormalities were profound with impaired re-epithelialization and granulated tissue formation in $\mathrm{KO}$ mice compared to those of WT mice. Morphometric analyses of wound sections (Fig. 1c-h) displayed a delay in wound closure in $\mathrm{KO}$ mice by quantification of the distance of newly formed epidermis covering the wound, an average of $39.74 \%$ of the wound was covered by epidermis in control mice, while only $29.40 \%$ of the wound was covered in Capns1-KO mice at the same timepoint (Fig. 1c, g, h). Likewise, the length of the new formed epithelium was significantly decreased $(1.65 \pm 0.13 \mathrm{~mm}$ in $\mathrm{KO}$ vs. $2.71 \pm 0.34 \mathrm{~mm}$ in WT mice) (Fig. 1d, g, h). These results suggest that wound healing is substantially delayed as a result of calpain- $1 / 2$ disruption in endothelial cells.

\section{Deletion of calpain in endothelial cells hinders the wound inflammatory response and angiogenesis}

The inflammatory phase is the inevitable stage of skin wound healing. To explore the potential role of calpain-1/2 in this inflammatory phase, we compared inflammatory cytokines from peripheral blood and infiltration of inflammatory cells in skin sections from 7-day-old excisional wounds of WT and KO mice. Serum TNF- $\alpha$ and IL6 levels were significantly higher in the WT mice than KO mice (TNF- $\alpha$ : $8.06 \pm 0.20 \mathrm{pg} / \mathrm{mL}$ in KO vs. $16.98 \pm 1.93 \mathrm{pg} /$ $\mathrm{mL}$ in WT mice, IL-6: $1.175 \pm 0.28 \mathrm{pg} / \mathrm{mL}$ in $\mathrm{KO}$ vs. $12.16 \pm 1.53 \mathrm{pg} / \mathrm{mL}$ in WT mice) (Fig. 2a, b). There was no significant difference in IL-10 serum levels between KO and WT mice (Fig. 2c). We also observed reduced numbers of $\mathrm{CD}^{+} \mathrm{T}$ lymphocytes and $\mathrm{CD} 68^{+}$macrophages in the wound beds of Capns1-KO mice compared to those of WT mice (Fig. $2 \mathrm{~d}-\mathrm{f}$ ), but no changes in the number of $\mathrm{CD}^{+} \mathrm{T}$ lymphocytes or $\mathrm{CD}_{4} 5^{+}$neutrophils (data not shown). These data indicate that deletion of calpain in endothelial cells reduces the inflammatory response in wounds.

CD31 staining of wound sections revealed a significant reduction in blood vessel density in $\mathrm{KO}$ relative to WT mice (Fig. 3a, b). Inflammatory cells are known to produce growth factors, cytokines, and chemokines that can act on vascular endothelial cells and can upregulate expression of adhesion molecules including ICAM-1 and VCAM-1. Consistent with the observed reduction in inflammatory cytokine expression, the expression of these endothelial adhesion molecules was reduced in wounds of $\mathrm{KO}$ relative to WT mice (Fig. 3a, c, d). Given the role of adhesion molecules in leukocyte recruitment, it is possible that the observed reduction in recruitment of $\mathrm{CD}^{+}$and $\mathrm{CD} 68^{+}$ cells is associated with reduced endothelial cell adhesion molecule expression. Together, these findings indicate that endothelial cell expression of calpain-1/2 promotes skin wound healing, possibility through effects on endothelial cell proliferation and inflammatory functions.

\section{Inhibition of calpain prevents inflammatory response to TNF-a in HUVECs}

Angiogenesis and inflammation are closely integrated processes in a number of physiological and pathological conditions $^{21,22}$. Inflammatory cells can directly release angiogenic factors, such as VEGF, ANG, bFGF, TGF- $\beta$, and 


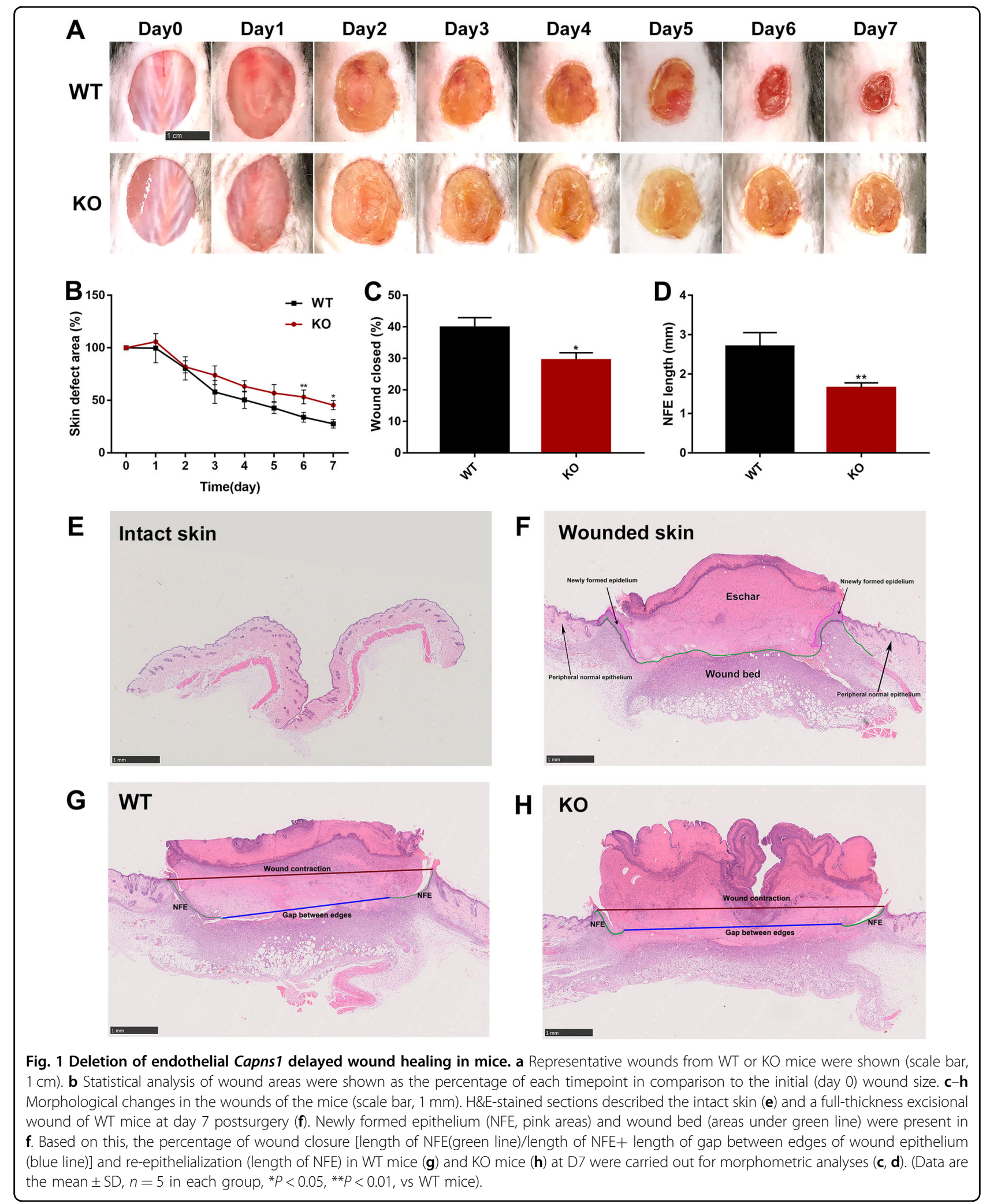

TNF- $\alpha$, thereby exerting mitogenic and migratory effects on the endothelium ${ }^{23,24}$. TNF- $\alpha$, as the main stimulus to mimic the inflammation environment in host tissue, may increase intracellular calcium concentration in macrophages and cardiomyocytes ${ }^{25,26}$. To explore the role of calpain on endothelial cell activation induced by 

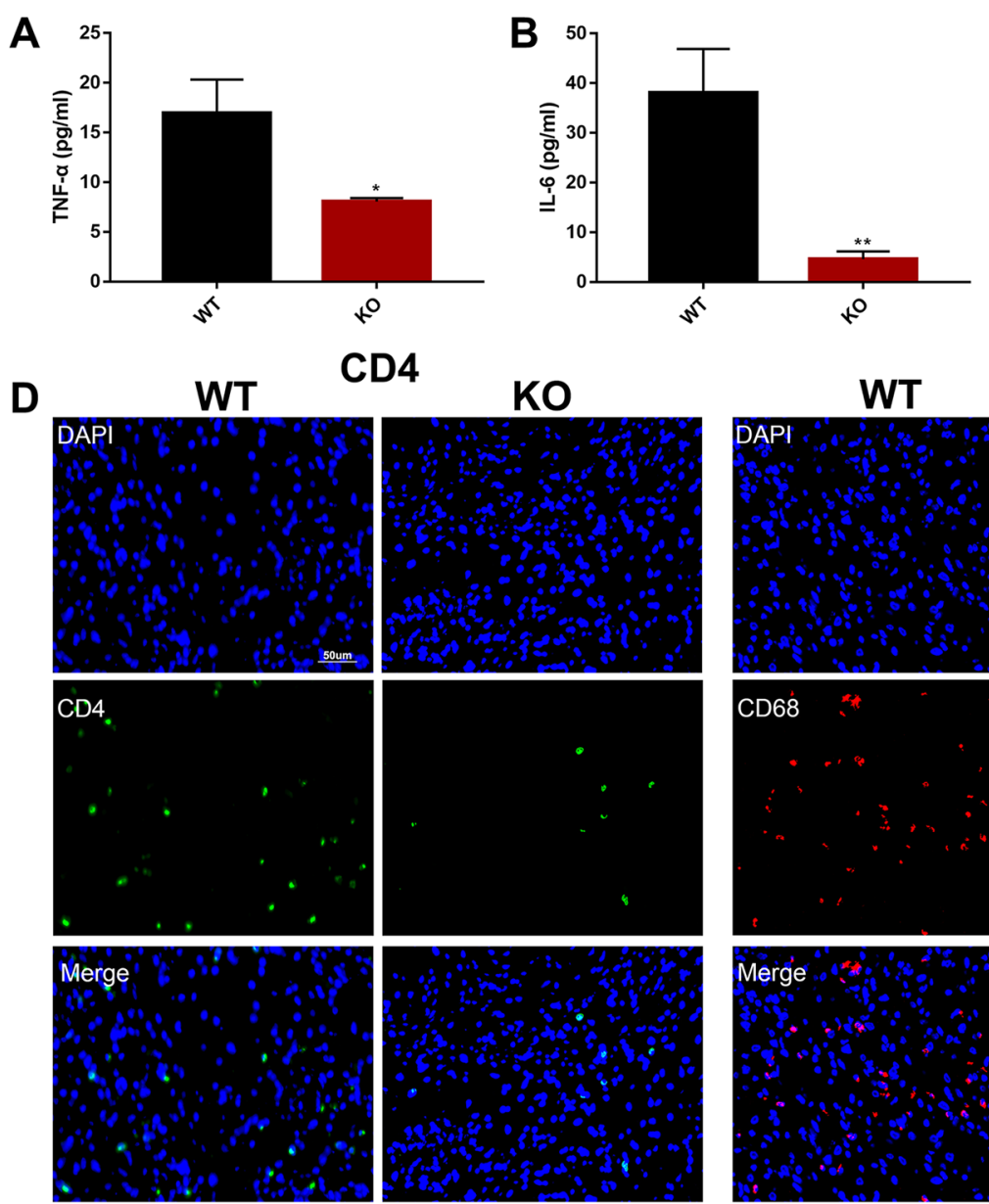

E

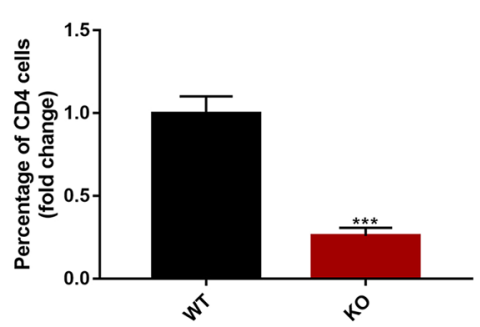

KO
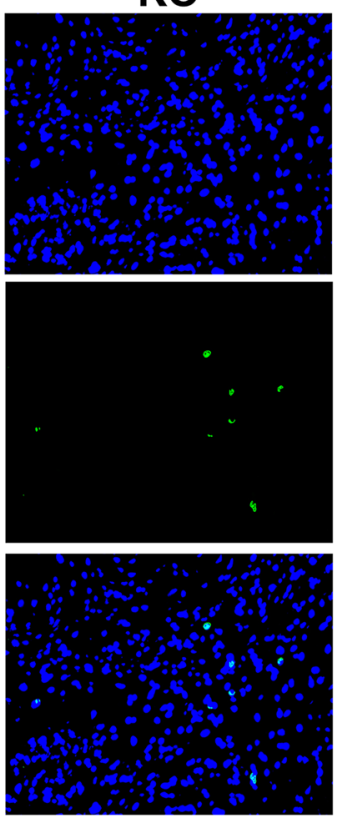

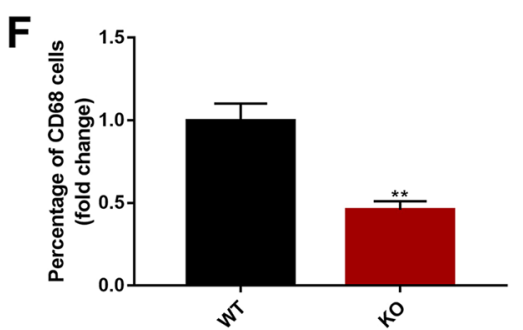

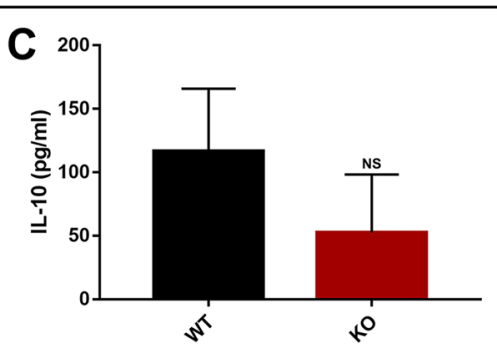

CD68
KO
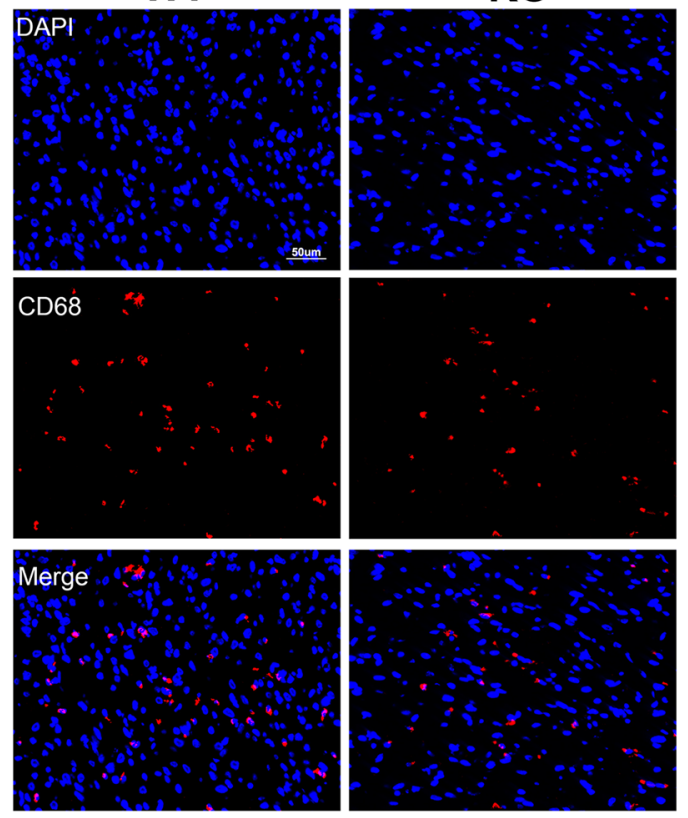

Fig. 2 Deletion of endothelial Capns 1 prevented the inflammatory responses in skin wounds. a-c The levels of serum TNF- $a$, IL-6, and IL-10 of mice at day 7 after injury were detected by ELISA. d, e Immunofluorescence images showing CD4 ${ }^{+} \mathrm{T}$ lymphocyte (gree) and CD68 ${ }^{+}$macrophage (red) infiltration in the wound beds. (scale bar, $50 \mu \mathrm{m}$ ). e, f Quantitative data of cell infiltration (data are the mean $\pm S D n=5$ in each group $* P<0.05$, ${ }^{*} P<0.01,{ }^{* * *} P<0.001$, vs WT mice).

proinflammatory factors, we infected human umbilical vascular endothelial cells (HUVECs) with adenovirus expressing the calpain-1/2 inhibitor CAST or bgalactosidase (Ad-CAST or Ad-gal, respectively), and then incubated the cells with TNF- $\alpha(10 \mathrm{ng} / \mathrm{mL})$ to mimic inflammation in vitro or vehicle for $6 \mathrm{~h}$ (Fig. 4a, b). Calpain activity was assessed by the cleavage of endogenous spectrin (a well-characterized calpain substrate), and quantified in cell lysates using the fluorescent substrate Ac-LLY-AFC. TNF- $\alpha$ treatment enhanced calpain activity (Fig. $4 b-d$ ), and stimulated the expression of the adhesion molecules ICAM-1 and VCAM-1 in HUVECs (Fig. 4c, e, f). Overexpression of calpastatin by Ad-CAST transfection inhibited calpain activity and activation of endothelial cells.

\section{Inhibition of calpain prevents proliferation and} angiogenesis in HUVECs under inflammatory conditions

Immune cells circulating though the microvasculature and extravasating into inflamed tissue contribute to angiogenesis by inducing migration, survival, and 

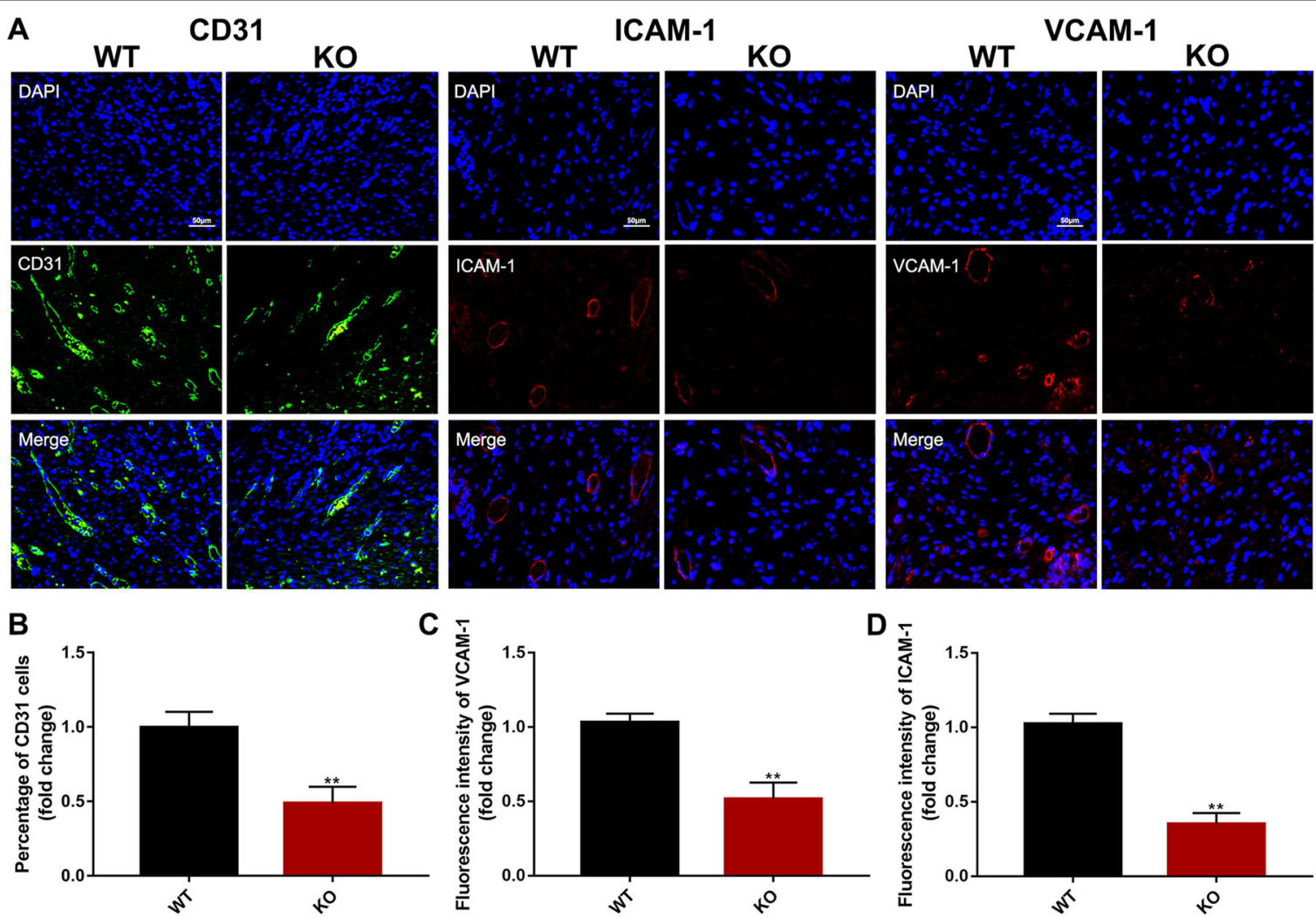

Fig. 3 Calpain promoted angiogenesis and endothelial cell activation at skin wound sites. a Seven days after injury, the wound sites were removed. Endothelial activation and vessel density were assessed by immunolabelling CD31 (marker of endothelial cells) (green) and ICAM-1 and VCAM-1 (markers of activated endothelial adhesion molecules) (red). $\mathbf{b}-\mathbf{d}$ Quantification of CD $31^{+}$cells, and expression of ICAM-1 and VCAM-1 in skin wounds. ( $n=5{ }^{* *} p<0.01$, vs WT mice).

proliferation of endothelial cells during the formation of new vasculature ${ }^{27}$. To investigate whether endothelial cell response to inflammatory stimulation was regulated by calpain, we assessed cell viability and proliferation (using CCK-8 and EdU assays, respectively), as well as migration and angiogenesis (using transwell or wound healing and tube formation assays, respectively) in HUVECs infected with Ad-CAST or Ad-gal after TNF- $\alpha(10 \mathrm{ng} / \mathrm{mL})$ or vehicle treatment for $6 \mathrm{~h}$. TNF- $\alpha$ promoted the proliferation, migration, and tube formation in HUVECs, but these effects were attenuated by infection with Ad-CAST (Figs. 5, 6). Consistently, incubation with Calpain inhibitor I (ALLN) achieved similar negative effects in HUVECs (Supplementary Figs. 2, 3). These results demonstrate that calpain promotes angiogenesis behavior of endothelial cells in response to TNF- $\alpha$-induced inflammation signaling.

\section{CAST overexpression enhanced ІкB expression and suppressed $\beta$-catenin protein in endothelial cells}

Calpain has been reported to cleave $\beta$-catenin and activate nuclear factor kappa-B (NF- $\mathrm{KB})$ through the proteolysis of $I_{\kappa} B$ in the context of angiogenesis and inflammation ${ }^{28,29}$. We therefore examined the effect of CAST overexpression on $\beta$-catenin and $N F-\kappa B$ in HUVECs challenged with TNF- $\alpha$. We showed that TNF- $\alpha$ induced $\beta$-catenin and suppressed ІкB expression in HUVECs, and these effects were both attenuated by infection with Ad-CAST (Fig. 7).

\section{Discussion}

In the present study, we demonstrated a protective role of endothelial calpain-1/2 during skin wound healing. In endothelial-specific Capns1-KO mice, wound healing was strikingly delayed (Fig. 1). During re-epithelialization, the density of the inflammatory infiltrates and blood vessels were reduced in $\mathrm{KO}$ mice. This was in accordance with in vitro data showing that calpain inhibition led to a reduction in proliferation, migration, and tube formation in HUVECs in response to proinflammation induction with TNF- $\alpha$. The deleterious effects of calpain deletion were associated with an upregulation of IкB and the downregulation of $\beta$-catenin during wound repair.

CAPNS1 acts as an obligate regulatory subunit for calpain- 1 and calpain- 2 by forming a heterodimer with their respective unique $80 \mathrm{kDa}$ large catalytic subunits ${ }^{30-32}$. 

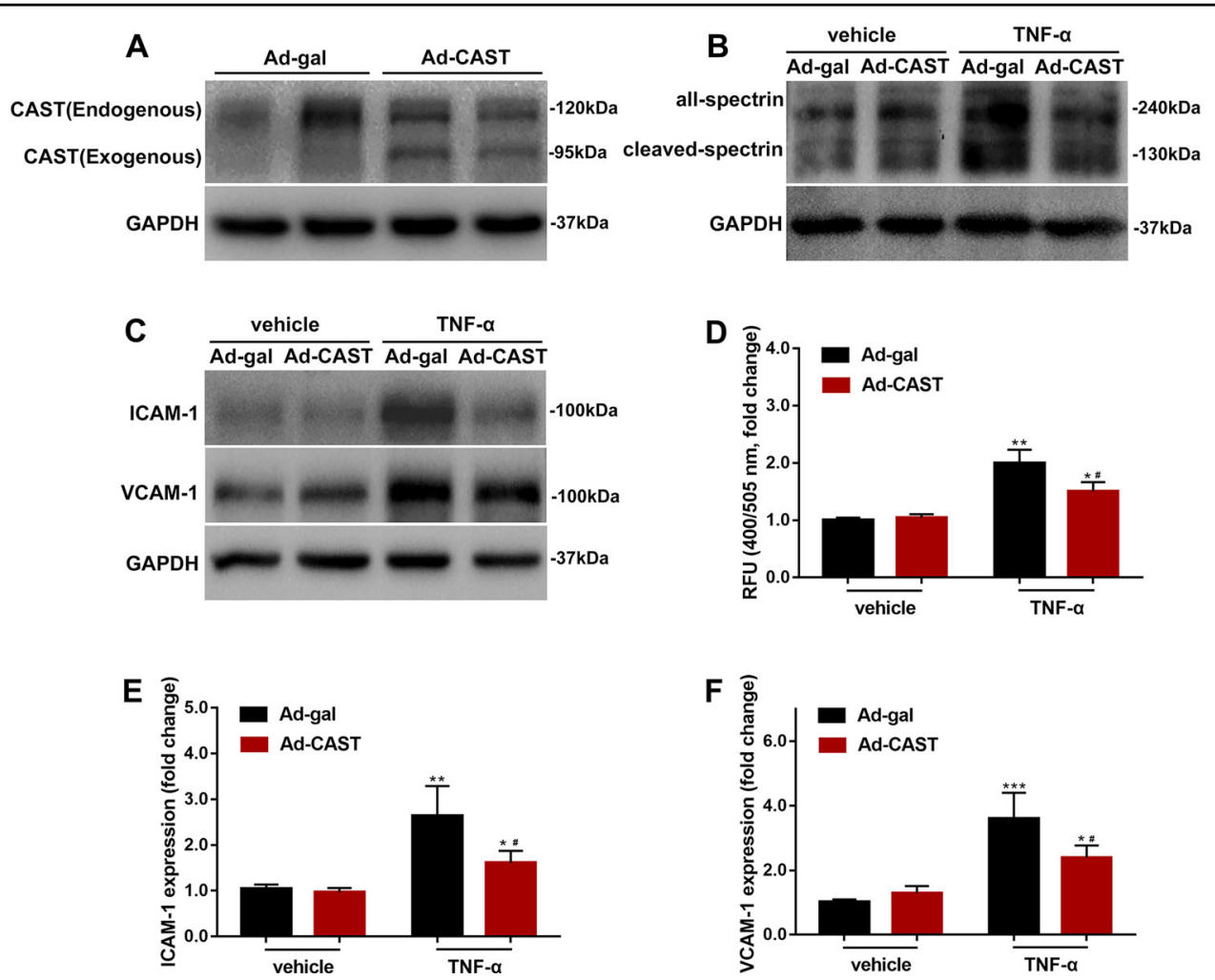

Fig. 4 Transfection of Ad-CAST inhibited the endothelial cell response to TNF-a stimulation. Cultured HUVECS were infected with Ad-CAST or Ad-gal as a control. After treatment with TNF-a $(10 \mathrm{ng} / \mathrm{mL})$ for $6 \mathrm{~h}$, the protein levels of calpastatin, all Spectrin, ICAM-1, VCAM-1, and GAPDH were determined by western blotting. a-c Representative western blots were shown. $\mathbf{d}$ Calpain activity was elevated as measured a fluorescence substrate, AC-LLY-AFC. e ICAM-1 protein levels relative to GAPDH. $\mathbf{f}$ VCAM-1 protein levels relative to GAPDH. (data are the mean $\pm S D, n=5$ independent cell batches. ${ }^{*} P<0.01$, ${ }^{* *} P<0.001$, vs Ad-gal + vehicle; ${ }^{\#} P<0.05$, vs Ad-gal + TNF- $a$ ).

Studies have shown that Capns1 expression is associated with oncogenesis in solid tumors ${ }^{32,33}$. Capns1 knockdown or knockout in breast carcinoma cells results in markedly decreased migration and invasion in vitro and metastatic activity in vivo in preclinical mouse models ${ }^{9,10,34}$. Other studies showed that calpain activity in endothelial cells was induced by VEGF, IL-6, or shear stress, and was involved in angiogenesis in skin wound healing ${ }^{18,35}$. Although the exact mechanisms by which calpain in endothelial cells improves repair of wounded skins are currently unknown, our findings suggest that roles in endothelial cell inflammatory signaling, and improved angiogenic responses may be important in this scenario.

Vascular ECs are located on the surface of the vascular endothelium, forming a barrier between circulating blood and local tissues. When ECs are damaged or exposed to inflammatory signals, such as TNFs and endotoxins, a series of metabolic and structural changes occur. This process is called endothelial cell activation. Activated endothelial cells increase the secretion of many cytokines, such as IL-1, IL-6, IL-10, and platelet activating factor, and the expression of cell surface adhesion molecules, including ICAM-1 and
VCAM-1. These molecules induce circulating inflammatory cells to roll along, adhere to and penetrate the endothelial surface, and further migrate into local tissues. Recruited leukocytes can promote angiogenesis by release of endothelial growth factors ${ }^{36,37}$. Thus, inflammation is often associated with increased angiogenesis.

Prior experiments and our data showed that sustained inflammation activates vascular endothelial cells, leading to sustained high expression of adhesion molecules and chemokines $^{38}$. Inflammation including infiltration of $\mathrm{CD} 4^{+} \mathrm{T}$ lymphocytes and $\mathrm{CD} 68^{+}$macrophages into the wound sites and high levels of serum TNF- $\alpha$ and IL- 6 were shown in the skin wound model (Fig. 2), accompanied by remarkable increases of adhesion molecules and $\mathrm{CD} 1^{+}$vessel density (Fig. 3). Neutrophils are the first immune cells to the wound bed and remain for about $24 \mathrm{~h}$ before undergoing apoptosis, helping to control infection and remove debris after tissue injury ${ }^{39,40}$. Cytokines released by neutrophils during apoptosis are chemotactic for monocytes and lymphocytes. These monocytes differentiate into macrophages, which can remain for several weeks at the wound site. Macrophages secrete 

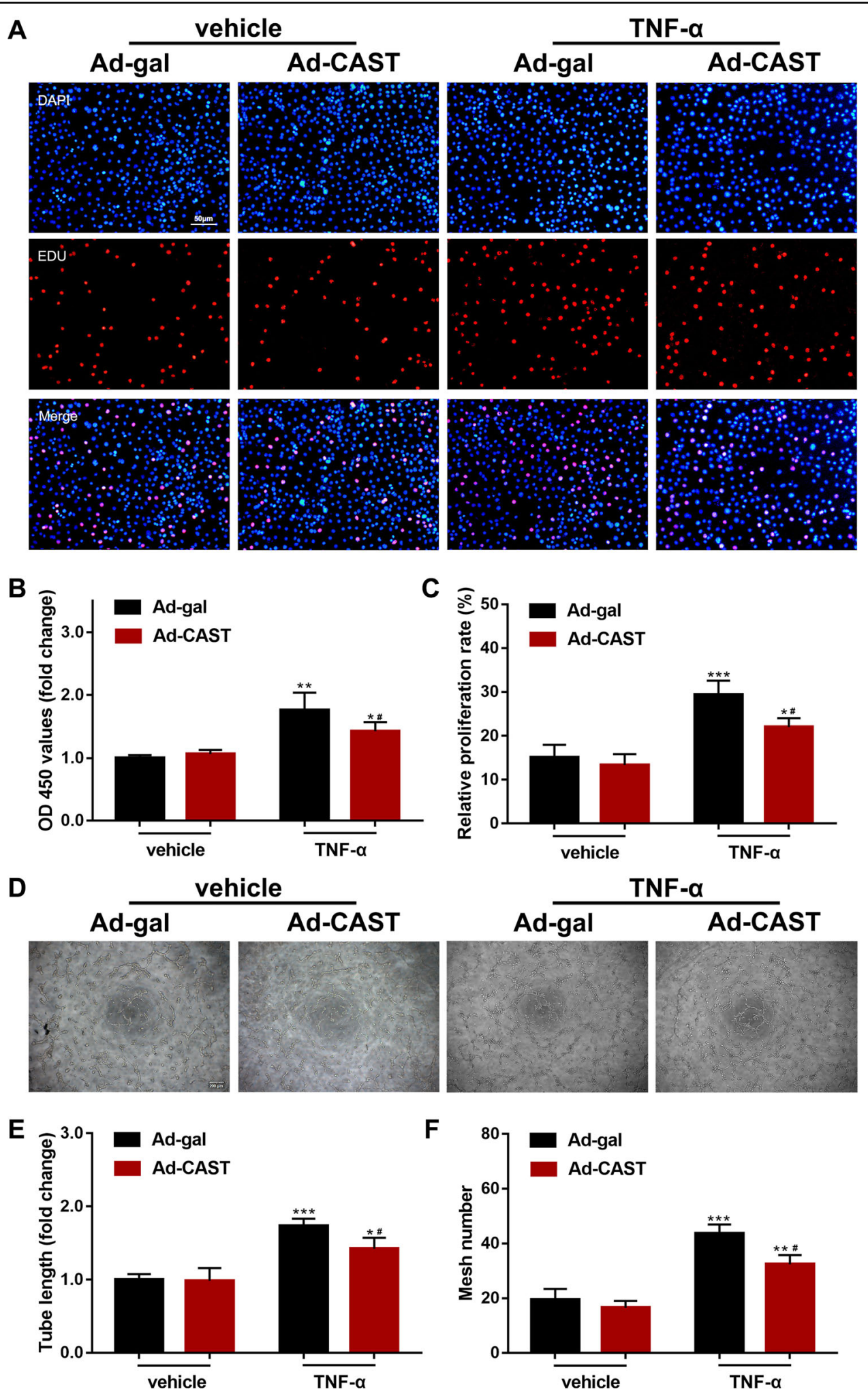

Fig. 5 Overexpression of CAST impaired cell viability, proliferation, and tube formation. Cultured HUVECs were infected with Ad-CAST or Ad-gal as a control in the presence of TNF-a $(10 \mathrm{ng} / \mathrm{mL}$ ) or vehicle. a A representative micrograph of endothelial proliferation via EdU assay (nuclear staining with DAPI, blue; proliferating cells stained with EdU, red) (scale bar, $50 \mu \mathrm{m})$. b Cell viability was detected by CCK-8 assay. c Statistical results for the EdU assay. d A representative micrograph for tube formation (scale bar, $200 \mu \mathrm{m}$ ). e, $\mathbf{f}$ Quantitative data of endothelial cell tube formation, as determined by tube length and mesh number (data are the mean $\pm S D, n=5$ independent cell batches. ${ }^{* * *} P<0.001$, vs Ad-gal + vehicle; ${ }^{\#} P<0.05$, vs Ad-gal + TNF-a). 


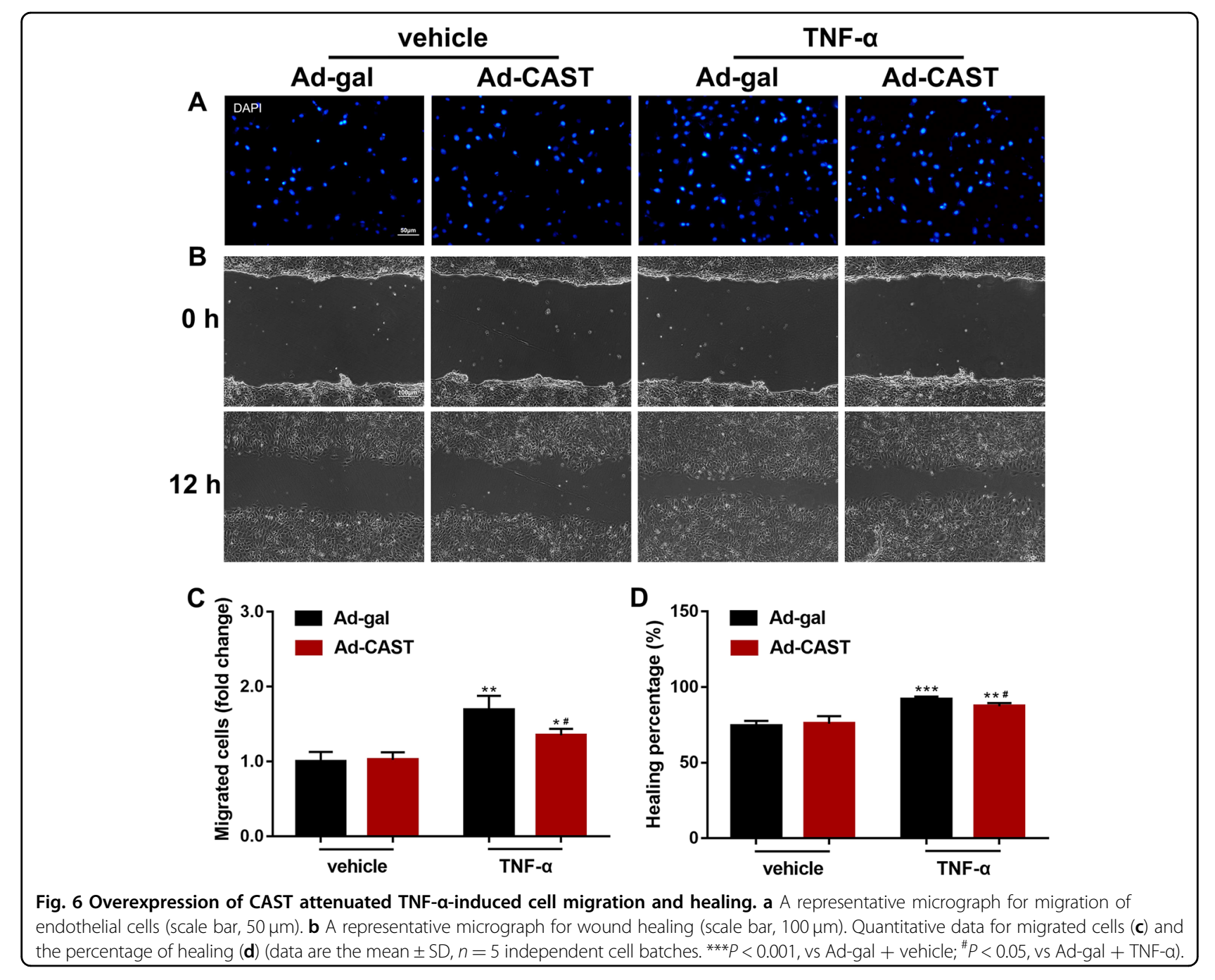

chemokines to attract $\mathrm{T}$ cells to the wound site and encourages $\mathrm{CD}^{+} \mathrm{T}$ helper polarization ${ }^{41}$. Apoptotic neutrophils are phagocytosed by the newly arrived macrophages. This could explain why reduced $\mathrm{T}$ lymphocytes and macrophages were observed in 7-day-old excisional wounds of $\mathrm{KO}$ mice, but neutrophil numbers were unaffected in this study.

Using an in vitro model, we showed that the inflammatory factor TNF- $\alpha$ enhanced ICAM- 1 and VCAM-1 expression, and promoted HUVECs proliferation, migration, and tube formation via activated calpain- $1 / 2$ activity (Figs. 4, 5). Inhibition of calpain blocked these proangiogenic phenotypes, in accordance with calpain participating in the activity and motility of endothelial cells ${ }^{42}$. NF- $\mathrm{KB}$ is an important nuclear transcription factor in the acute and chronic inflammatory response. Inhibition of NF- $\mathrm{KB}$ prevents the activation of endothelial cells though the overexpression of IкB, an endogenous inhibitor of NF- $\mathrm{KB}^{43,44}$. Calpains have also been reported to be modulators of the $\mathrm{NF}-\mathrm{kB}$ signaling pathway via the degradation of $\mathrm{I} \kappa \mathrm{B}^{45}$, which was verified in our experiments. Thus, endothelial calpain-1/2 might contribute to inflammation through effects on the NF- $\mathrm{kB}$ signaling pathway.

Endothelial calpain systems are also responsible for angiogenic regulation. Our previous study showed that deletion of endothelial cell Capns1 in mice reduced diabetic cardiomyopathy by improving angiogenesis induced by increased $\beta$-catenin protein levels ${ }^{18}$, which is consistent with the $\beta$-catenin-mediated improvement in angiogenesis in several studies ${ }^{29,46}$. In contrast, the present study demonstrated that endothelial calpain activity promotes angiogenesis in skin wound repair and HUVECs under TNF- $\alpha$ stimulation. Intriguingly, calpain-1/2 inhibition reduced the TNF- $\alpha$-induced protein level of $\beta$-catenin. This mechanism may be related to crosstalk between $\mathrm{Wnt} / \beta$-catenin and the NF- $\mathrm{kB}$ signaling pathways during inflammation. Several studies have reported a positive regulation of $\mathrm{Wnt} / \beta$-catenin signaling by the NF- $\mathrm{BB}$ pathway. IKK $\alpha$ and IKK $\beta$, the critical activators of the NF- $\mathrm{KB}$ pathway, are also 


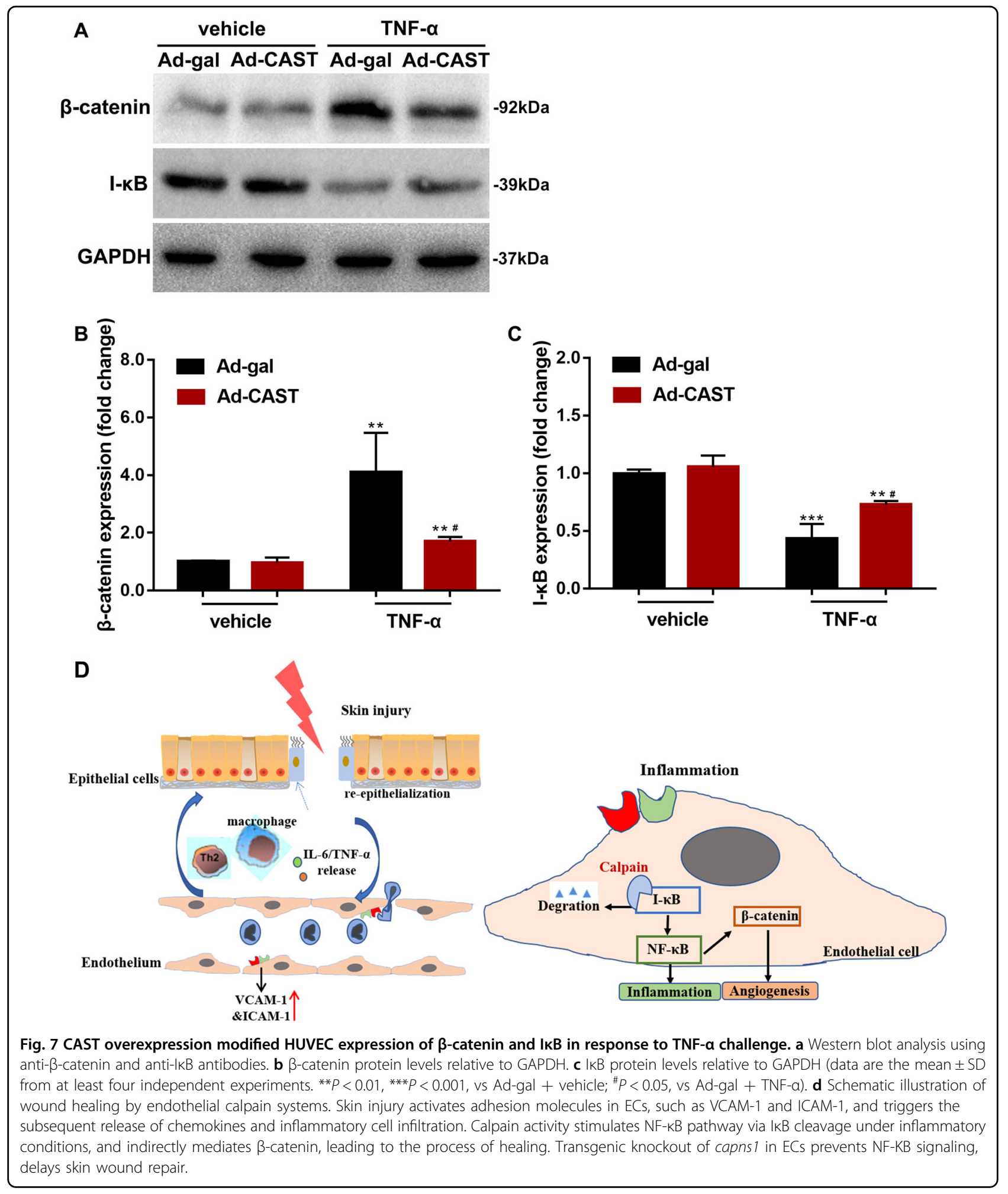

involved in the regulation of $\beta$-catenin-dependent transcriptional activity ${ }^{47-49}$. Some studies support that calpain activity is required for wound healing, transgenic mice with overexpressed CAST, which displayed a striking delay in skin wound healing through impaired angiogenesis ${ }^{13,50}$. These differences imply that calpain$1 / 2$ modulates angiogenesis via multiple mechanisms, depending on the environment and tissues. However, 
the underlying molecular mechanisms of this finding remain to be elucidated.

In conclusion, our study sheds new light on the roles of endothelial calpain in skin wound healing through inflammation-induced angiogenesis. Calpain-1/2 inhibition impedes inflammatory cell recruitment and angiogenesis, resulting in delayed wound healing and cell injury. Therefore, the effects of modifying calpain activity on inflammation, wound healing and skin regeneration should be carefully considered in the context of future therapeutic applications.

\section{Materials and methods}

\section{Animals}

All experimental procedures were approved by the Animal Use Subcommittee of Soochow University (Suzhou, China). C57BL/6 mice and TEK-CRE ${ }^{+/-}$mice were purchased from Jackson Laboratory (Sacramento, CA, USA). Mice with endothelial cell-specific Capns1 knockout (KO) were generated by breeding Capns1 floxed $\left(\right.$ Capns $1^{\mathrm{PZ} / \mathrm{PZ}}$ ) and TEK-CRE ${ }^{+/-}$mice as previously described $^{18}$. The breeding program was implemented at the animal care facilities of Soochow University's.

Genotyping was detetmined by PCR, using gene-specific primers (Supplementary Table1 and Supplementary Fig. 1).

\section{Dorsal excisional wound model}

The mice were anesthetized by inhalation of isoflurane, and full-thickness excisional skin wounds were made in the dorsal skin using a sterile biopsy punch with a diameter of $15 \mathrm{~mm}$ (Kai Industries, Tokyo, Japan). Wounds were left uncovered until they were harvested. After surgery, the dorsal wounds were photographed every day using a Sony CybershotH 10.1 megapixel DSC-W180 digital camera. Changes in wound areas were expressed as the proportion of the initial wound areas. Subsequently, the wounds and their surrounding areas, including the scab and epithelial margins, were harvested for further analysis. All quantifications were performed on tissue samples obtained from males only. The experimenters were not blinded and were aware of the genotype of the animals prior to experimentation. No statistical method was applied to predetermine the same size of experimental groups. Appropriate animal health status, in accordance with our animal experimentation protocol, was used for exclusion criteria. No randomisation of the animals was used as experimental group assignment was primarily dependent on the appropriate animal genotype.

\section{Histological analysis}

On the 7th day after injury, skin samples were obtained and fixed in $4 \%$ paraformaldehyde overnight, and then embedded in paraffin. Paraffin blocks were processed into
5- $\mu \mathrm{m}$ thick sections. Hematoxylin and eosin (H\&E) staining was performed according to standard protocols, and morphometric analysis was conducted as previously described $^{51}$. For immunofluorescence, protocols that are described elsewhere were applied ${ }^{52}$. Briefly, sections were deparaffined and incubated with primary antibody overnight at $4{ }^{\circ} \mathrm{C}$ after blocking with $5 \%$ BSA, and then visualized using secondary antibodies in blocking buffer for $1 \mathrm{~h}$ at room temperature. The primary monoclonal antibodies included anti-CD4, CD8, CD45, CD68, CD31, intercellular adhesion molecule-1 (ICAM-1) and vascular adhesion molecule-1 (VCAM-1) (Thermo Scientific, USA), AlexaFluor-488 -conjugated IgGs (Invitrogen, USA) secondary antibodies were used. DAPI (Beyotime, China) is used as a nuclear counterstain at a working concentration of $1 \mu \mathrm{g} / \mathrm{mL}$. Immunofluorescence-stained and H\&E-stained sections were imaged using a DMI 6000B microscope (Leica, Germany). Image analysis and quantifications were performed using ImageJ (National Institutes of Health, USA).

\section{Morphometric analysis}

Morphometric analysis was performed on $5 \mu \mathrm{m}$ sections obtained from the middle of the D7 wounds and were stained with H\&E. Measurements of wound closure, HPE area (area of the epidermis measured from the point outside of the wound, at which it starts to become thicker than normal epithelium), and the length of newly formed wound epithelium (re-epithelialization) were carried out using ImageJ as previously described ${ }^{20}$.

\section{Measurement of leukocyte numbers and neovascularization at wound sites}

The numbers of infiltrating $\mathrm{CD} 4^{+}, \mathrm{CD} 8^{+}, \mathrm{CD} 45^{+}$and $\mathrm{CD} 68^{+}$cells, and CD31 ${ }^{+}$ECs within the wound beds were enumerated in five randomly chosen visual fields of the sections, and the average of the selected five fields was calculated.

\section{ELISA measurement of serum TNF- $a$, IL- 6 , and IL-10 levels}

The serum from the wounded mice was obtained, and TNF- $\alpha$, IL-6, and IL-10 levels were measured by commercially available ELISA kits (Beyotime, China) according to the manufacturer's recommendation.

\section{Cell culture and adenoviral infection}

Human umbilical vein endothelial cells (HUVECs) were purchased from Lonza (Verviers, Belgium) and cultured in ECM (ScienCell Research Laboratories, USA) containing $10 \%$ fetal bovine serum (FBS) at $5 \% \mathrm{CO}_{2}$, and $37^{\circ} \mathrm{C}$. Cells were used for experiments up to passage number 5 . HUVECs were infected with adenoviral vectors containing rat calpastatin ([Ad-CAST] University of Buffalo, Buffalo, USA) or $\beta$-galactosidase ([Ad-gal] Vector Biolabs) 
at a multiplicity of infection of 100 plaque-forming units/ cell. All experiments were performed after $24 \mathrm{~h}$ of adenoviral infection.

\section{Western blot analysis}

HUVECs were collected and lysed with RIPA lysis buffer after treatments. The protein concentration was detected using a BCA kit (Beyotime, China). The protein levels of spectrin, ICAM- 1 , VCAM- $1, \beta$-catenin, IкB, and GAPDH (1:1000 dilutions, Cell Signaling Technology, USA) were determined by western blotting. Blots were scanned and quantified using an Odyssey imaging system (LI-COR Biosciences, USA). Quantified band intensities were normalized using GAPDH.

\section{Calpain activity assay}

Calpain activity was assayed using a fluorometric kit (Abcam, Cambridge, UK) according to the manufacturer's protocol. Calpain activity was analyzed using a fluorometer equipped with a 400-nm excitation filter and 505nm emission filter.

\section{Cell viability and proliferation assays}

Cell viability and proliferation were determined by using a CCK-8 kit (Kumamoto, Japan) and an EdU staining kit (RiboBio, China), respectively. Briefly, HUVECs were infected with Ad-CAST or Ad-gal, and then seeded onto culture plates. After treatment with $10 \mathrm{ng} / \mathrm{mL}$ TNF- $\alpha$ (Lonza, Verviers, Belgium) for $6 \mathrm{~h}$, the absorbance was detected at $450 \mathrm{~nm}$ according to manufacturer instructions for the CCK-8 kit. For the EdU assay, the proliferation of HUVECs was detected with the Cell-Light EdU Apollo 488 In Vitro Imaging Kit (RiboBio, China) according to the manufacturer's protocol. Briefly, HUVECs were seeded into 12 -well plates at the density of $5 \times 10^{4} /$ well. After various treatments, the cells were stained with $50 \mu \mathrm{mol} / \mathrm{L} \mathrm{EdU} \mathrm{for}$ $2 \mathrm{~h}$, fixed with $4 \%$ paraformaldehyde, and incubated with $2 \mathrm{mg} / \mathrm{mL}$ glycine for $5 \mathrm{~min}$ and $0.5 \%$ Triton X-100 in PBS for $10 \mathrm{~min}$. Then, the cells were stained with $5 \mu \mathrm{g} / \mathrm{mL}$ DAPI and manually counted in five random high power fields $(\times 100)$ in each well.

\section{Transwell assay}

Migration analyses were carried out using the transwell chambers, which included upper and lower chambers separated by a polycarbonate filter $(8 \mu \mathrm{m}$ pore size). HUVECs were collected and resuspended in ECM without fetal bovine serum (FBS). Cells were seeded at $1 \times 10^{4}$ cells $/ 100 \mu \mathrm{L}$ in the upper compartments of a 24-well plate, and $500 \mu \mathrm{L}$ of medium containing 10\% FBS was added into the lower chamber. The chambers were incubated at $37^{\circ} \mathrm{C}$, in $5 \% \mathrm{CO}_{2}$ for $12 \mathrm{~h}$. Cells in the lower chamber were stained with DAPI, and manually counted in five random high power fields $(\times 200)$ in each well.

\section{Wound-healing assay}

HUVECs were seeded in 12-well plates and cultured until at least $90 \%$ confluence. Wounds were made with a sterile pipette tip. After the dislodged cells were washed away, the cells were recultured with FBS-free media. Wounds were photographed at the initial timepoint and $12 \mathrm{~h}$ postwounding at the same location. The extent of healing was defined as the difference between the original and remaining wound areas, and is expressed as a percentage of the original area.

\section{Tube formation assay}

The angiogenic ability of HUVECs was assessed by a tube formation assay as described previously ${ }^{18}$. Tube formation was determined by measuring tube length and mesh number.

\section{Statistical analysis}

All data were analyzed by using Prism 7.0 software. One-or two-way ANOVA followed by Newman-Keuls tests were performed for multigroup comparisons, as appropriate. Student's $t$-test was used for comparisons between two groups. A value of $p<0.05$ was considered statistically significant.

\section{Acknowledgements \\ We thank Tianqing Peng for his advice in the experiments, and Peter A.Greer for his advice in the revised draft. \\ Author contributions \\ Conceived and designed the experiments: X.T., Z.S., and H.H. Performed the experiments: C.Y., X.T. and W.W. Analyzed the data: C.Y., X.T., and D.Z. Contributed reagents/materials/analysis tools: D.Z. Writing-original draft preparation: C.Y. and X.T.; Writing-review and editing: H.H., Z.S., and X.T. This study was financially supported from the National Natural Science Foundation of China [81700361, 91839101] and Introduction Project of Clinical Medicine Expert Team for Suzhou (No. SZYJTD201704). National Natural Science Foundation of China (91839101, 81700361); Introduction Project of Clinical Medicine Expert Team for Suzhou (No. SZYJTD201704).}

Conflict of interest

The authors declare that they have no conflict of interest.

\section{Publisher's note}

Springer Nature remains neutral with regard to jurisdictional claims in published maps and institutional affiliations.

Supplementary Information accompanies this paper at (https://doi.org/ 10.1038/s41419-020-02737-x).

Received: 6 December 2019 Accepted: 23 June 2020

Published online: 14 July 2020

\footnotetext{
References

1. Gurtner, G. C., Werner, S., Barrandon, Y. \& Longaker, M. T. Wound repair and regeneration. Nature 453, 314-321 (2008).

2. Johnson, A. \& Dipietro, L. A. Apoptosis and angiogenesis: an evolving mechanism for fibrosis. FASEB J. 27, 3893-3901 (2013).

3. Eming, S. A., Martin, P. \& Tomiccanic, M. Wound repair and regeneration: mechanisms, signaling, and translation. Sci. Transl. Med. 6, $265 \mathrm{sr} 6$ (2014).
} 
4. Lansdown, A. B. G. Calcium: a potential central regulator in wound healing in the skin. Wound Repair Regen. 10, 271-285 (2002).

5. Goll, D. E., Thompson, V. F., Li, H., Wei, W. \& Cong, J. The calpain system. Physiol. Rev. 83, 731-801 (2003)

6. Zhang, Y., Liu, N. M., Wang, Y., Youn, J. Y. \& Cai, H. Endothelial cell calpain as a critical modulator of angiogenesis. Biochim. Biophys. Acta 1863, 1326-1335 (2017).

7. Cuzzocrea, S., McDonald, M. C., Mazzon, E., Siriwardena, D. \& Thiemermann, C. Calpain inhibitor I reduces the development of acute and chronic inflammation. Am. J. Pathol. 157, 2065-2079 (2001).

8. Storr, S. J., Carragher, N. O., Frame, M. C., Parr, T. \& Martin, S. G. The calpain system and cancer. Nat. Rev. Cancer 11, 364-374 (2011).

9. Grieve, S., Gao, Y., Hall, C., Hu, J. \& Greer, P. A. Calpain genetic disruption and HSP90 inhibition combine to attenuate mammary tumorigenesis. Mol. Cell. Biol. 36, 2078-2088 (2016).

10. Macleod, J. A. et al. Genetic disruption of calpain-1 and calpain-2 attenuates tumorigenesis in mouse models of HER2 + breast cancer and sensitizes cancer cells to doxorubicin and lapatinib. Oncotarget 9, 33382-33395 (2018).

11. Liang, B., Duan, B., Zhou, X., Gong, J. \& Luo, Z. Calpain activation promotes BACE1 expression, amyloid precursor protein processing, and amyloid plaque formation in a transgenic mouse model of Alzheimer disease. J. Biol. Chem. 285, 27737-27744 (2010).

12. Ni, R. et al. Mitochondrial calpain-1 disrupts ATP synthase and induces superoxide generation in type 1 diabetic hearts: a novel mechanism contributing to diabetic cardiomyopathy. 65, 255-268 (2016).

13. Nassar, D., Letavernier, E., Baud, L., Aractingi, S. \& Khosrotehrani, K. Calpain activity is essential in skin wound healing and contributes to scar formation. PLOS ONE 7, e37084 (2012).

14. Lin, Z. et al. Loss-of-function mutations in CAST cause peeling skin, leukonychia, acral punctate keratoses, cheilitis, and knuckle pads. Am. J. Hum. Genet. 96, 440-447 (2015).

15. Liu, W. et al. The abnormal architecture of healed diabetic ulcers is the result of FAK degradation by calpain 1. J. Invest. Dermatol. 137, 1155-1165 (2017).

16. Miyazaki, T. \& Miyazaki, A. Dysregulation of calpain proteolytic systems underlies degenerative vascular disorders. J. Atheroscler. Thromb. 25, 1-15 (2018).

17. Chen, B. et al. Inhibition of calpain reduces oxidative stress and attenuates endothelial dysfunction in diabetes. Cardiovasc. Diabetol. 13, 88-88 (2014).

18. Teng, X. et al. Selective deletion of endothelial cell calpain in mice reduces diabetic cardiomyopathy by improving angiogenesis. Diabetologia $\mathbf{6 2}$, 860-872 (2019).

19. Ansell, D. M., Campbell, L., Thomason, H. A., Brass, A. \& Hardman, M. J. A statistical analysis of murine incisional and excisional acute wound models. Wound Repair Regen. 22, 281-287 (2014).

20. Kumin, A. et al. Peroxiredoxin 6 is required for blood vessel integrity in wounded skin. J. Cell Biol. 179, 747-760 (2007).

21. Benelli, R., Lorusso, G., Albini, A. \& Noonan, D. M. Cytokines and chemokines as regulators of angiogenesis in health and disease. Curr. Pharm. Des. 12, 3101-3115 (2006).

22. Schon, M. P. \& Ludwig, R. Lymphocyte trafficking to inflamed skin-molecular mechanisms and implications for therapeutic target molecules. Expert Opin. Ther. Targets 9, 225-243 (2005).

23. Gong, R., Rifai, A. \& Dworkin, L. D. Anti-inflammatory effect of hepatocyte growth factor in chronic kidney disease: targeting the inflamed vascular endothelium. J. Am. Soc. Nephrol. 17, 2464-2473 (2006).

24. Lee, Y. C. The involvement of VEGF in endothelial permeability: a target for anti-inflammatory therapy. Curr. Opin. Investig. Drugs 6, 1124 (2005).

25. Tano, J. Y., Lee, R. H. \& Vazquez, G. Involvement of calmodulin and calmodulin kinase $\|$ in tumor necrosis factor alpha-induced survival of bone marrow derived macrophages. Biochem. Biophys. Res. Commun. 427, 178-184 (2012).

26. Wang, G., Wang, H., Yao, Y., Guo, L. \& Liu, P. The role of Ca2+/calmodulindependent protein kinase $\|$ and calcineurin in TNF-a-induced myocardial hypertrophy. Braz. J. Med. Biol. Res. 45, 1045-1051 (2012).

27. Costa, C., Incio, J. \& Soares, R. Angiogenesis and chronic inflammation: cause or consequence? Angiogenesis 10, 149-166 (2007).

28. Benetti, $\mathrm{R}$. et al. The calpain system is involved in the constitutive regulation of beta-catenin signaling functions. J. Biol. Chem. 280, 22070-22080 (2005).
29. Shen, Y. et al. Thromboxane governs the differentiation of adipose-derived stromal cells toward endothelial cells in vitro and in vivo. Circ. Res. 118, 1194-1207 (2016)

30. Saez, M. E., Ramirezlorca, R., Moron, F. J. \& Ruiz, A. The therapeutic potential of the calpain family: new aspects. Drug Discov. Today 11, 917-923 (2006).

31. Grahamsiegenthaler, K., Gauthier, S., Davies, P. L. \& Elce, J. S. Active recombinant rat calpain II. Bacterially produced large and small subunits associate both in vivo and in vitro. J. Biol. Chem. 269, 30457-30460 (1994).

32. Arthur, J. S., Elce, J. S., Hegadorn, C., Williams, K. \& Greer, P. A. Disruption of the murine calpain small subunit gene, Capn4: calpain is essential for embryonic development but not for cell growth and division. Mol. Cell. Biol. 20, 4474-4481 (2000).

33. Li, Y. et al. The oncoprotein HBXIP enhances migration of breast cancer cells through increasing filopodia formation involving MEKK2/ERK1/2/ Capn4 signaling. Cancer Lett. 355, 288-296 (2014).

34. Dai, Z. et al. Capn4 contributes to tumour growth and metastasis of hepatocellular carcinoma by activation of the FAK-Src signalling pathways. J. Pathol. 234, 316-328 (2014).

35. Zhang, Y., Li, Q., Youn, J. \& Cai, H. PTP1B in calpain dependent feedback regulation of VEGFR2 in endothelial cells: implication in VEGFdependent angiogenesis and diabetic wound healing. FASEB J. 292 407-416 (2016).

36. Schruefer, R., Lutze, N., Schymeinsky, J. \& Walzog, B. Human neutrophils promote angiogenesis by a paracrine feedforward mechanism involving endothelial interleukin-8. Am. J. Physiol. Heart Circ. Physiol. 288, H1186 (2005).

37. Mccourt, M., Wang, J. H., Sookhai, S. \& Redmond, H. P. Proinflammatory mediators stimulate neutrophil-directed angiogenesis. Arch. Surg. 134 1325-1331 (1999).

38. Pober, J. S. \& Sessa, W. C. Evolving functions of endothelial cells in inflammation. Nat. Rev. Immunol. 7, 803-815 (2007).

39. Brubaker, A. L., Schneider, D. F. \& Kovacs, E. J. Neutrophils and natural killer $\mathrm{T}$ cells as negative regulators of wound healing. Expert Rev. Dermatol. 6, 5-8 (2011).

40. Ellis, S., Lin, E. J. \& Tartar, D. Immunology of wound healing. Curr. Dermatol. Rep. 7, 350-358 (2018).

41. Larouche, J., Sheoran, S., Maruyama, K. \& Martino, M. M. Immune regulation of skin wound healing: mechanisms and novel therapeutic targets. Adv. Wound Care (New Rochelle) 7, 209-231 (2018).

42. Bodnar, R. J., Yates, C. C. \& Alan, W. IP-10 blocks vascular endothelial growth factor-induced endothelial cell motility and tube formation via inhibition of calpain. Circ. Res. 98, 617-625 (2006).

43. Cooper, J. T. et al. A20 Blocks Endothelial Cell Activation through a NF-KBdependent Mechanism. J. Biol. Chem. 271, 18068-18073 (1996).

44. Anrather, J., Csizmadia, V. C., Soares, M. P., Bach, F. H. \& Winkler, H. Inhibition of bovine endothelial cell activation in vitro by regulated expression of a transdominant inhibitor of NF-kappa B. J. Clin. Invest. 99, 763-772 (1997).

45. Shumway, S. D., Maki, M. \& Miyamoto, S. The PEST domain of IkappaBalpha is necessary and sufficient for in vitro degradation by mu-calpain. J. Biol. Chem. 274, 30874 (1999).

46. Li, G. \& lyengar, R. Calpain as an effector of the Gq signaling pathway for inhibition of Wnt/ $\beta$-catenin-regulated cell proliferation. Proc. Natl Acad. Sci. USA 99, 13254-13259 (2002)

47. Ma, B. \& Hottiger, M. O. Crosstalk between Wnt/B-catenin and NF-KB signaling pathway during inflammation. Front. Immunol. 7, 378 (2016).

48. Lamberti, $C$. et al. Regulation of $\beta$-catenin function by the IKB kinases. J. Biol. Chem. 276, 42276-42286 (2001).

49. Li, D. et al. Myeloid cell RelA/p65 promotes lung cancer proliferation through Wnt/beta-catenin signaling in murine and human tumor cells. Oncogene 33, 1239-1248 (2014).

50. Demidowich, A. P., Jun, J. Y. \& Yanovski, J. A. Polymorphisms and mutations in the melanocortin-3 receptor and their relation to human obesity. Biochim. Biophys. Acta 1863, 2468-2476 (2017).

51. Parfejevs, V. et al. Injury-activated glial cells promote wound healing of the adult skin in mice. Nat. Commun. 9, 236 (2018).

52. Dong, M. W. et al. Activation of a7nAChR promotes diabetic wound healing by suppressing AGE-induced TNF-a production. Inflammation 39, 687-699 (2016). 